\title{
Resource Competition and Coevolution in Sticklebacks
}

\author{
Dolph Schluter
}

Published online: 15 January 2010

(C) Springer Science+Business Media, LLC 2010

\begin{abstract}
Threespine stickleback in young postglacial lakes provide a compelling example of coevolution between species that compete for resources. Coexisting pairs of stickleback species are highly divergent in habitat, diet, and body size and shape, whereas stickleback occurring alone in lakes are intermediate. We used experiments in ponds to test mechanisms of divergence between coexisting species. The results support the hypothesis of coevolution by resource competition between stickleback, but we found evidence that interactions with natural enemies also contribute to divergence. Natural selection arising from these interactions selects against intermediate phenotypes, included hybrids, and thus has contributed to the origin and persistence of stickleback species.
\end{abstract}

Keywords Stickleback · Coevolution · Character displacement $\cdot$ Speciation $\cdot$ Natural selection

\section{Introduction}

I address the consequences of coevolution resulting from interspecific competition for resources-also known as "character displacement" (Brown and Wilson 1956) and "coevolutionary displacement" (Thompson 2005). By resource competition I mean the negative impact of one species on another resulting from consumption and depletion of shared resources. Competition occurs frequently between species in nature, but it is not as obvious as some of the other interactions discussed in this issue. Resource

\section{Schluter $(\triangle)$}

Biodiversity Research Centre and Zoology Department,

University of British Columbia,

Vancouver, BC V6T 1Z4, Canada

e-mail: schluter@zoology.ubc.ca competitors do not usually kill, eat, nourish, help, or live on or inside one another. Individuals of two species sharing a resource need not even meet to compete. Competition results indirectly from the changes each interacting species makes to its food supply in the environment. The interaction is thus not observed directly, but must be inferred from experiments or by careful measurements of food depletion and its impacts.

Competition has long been thought to be one of the most important interactions in nature, with widespread evolutionary consequences. Many of the naturalists studying evolution in the last century believed that this interaction was one of the main drivers of differentiation between closely related species. Darwin (1859) thought that competition was important in the very origin of new species. Without resource competition, it was believed, the breadth of life's diversity on earth would be much less than it actually is.

These are bold claims, but are they correct? Until recently, virtually all the evidence in favor came from observation of patterns in nature, such as the one illustrated in Fig. 1. Lack (1947) showed that beak sizes of species of Darwin's ground finches tend to be more different from one another when they occur on the same Galápagos island than when they occur on different islands. Beak size in these birds is related to the size and hardness of seeds that can be consumed (Grant and Grant 2008). Lack proposed that competition for seeds had caused the evolution of exaggerated differences in beak sizes between finch species that coexist. In contrast, when each species was alone on an island its beak size would converge to that of the other species to exploit a similar range of seed sizes. Brown and Wilson (1956) subsequently presented examples of the same pattern in other traits and species. Studies have continued to accumulate, and we now have dozens of examples from all kinds of organisms - beak sizes of birds, 
body size of snails, tooth sizes of weasels, flowering times of plants, body shape of fish, carnivory of tadpoles, and more (Schluter 2000). Mathematical theory has also indicated that, given the right conditions, divergence is expected to occur readily between competing species by a coevolutionary sequence of reciprocal changes in the traits used to consume resources (Taper and Case 1985; Abrams 1986b; Doebeli 1996). Together this data and theory suggested that resource competition between species really is an important process in evolution.

The trouble with patterns like those in Fig. 1, however, is that they don't get at the underlying mechanism. Compe- tition and coevolution provides a plausible explanation for trait differences between coexisting species, but unfortunately it is not the only explanation. Since Lack (1947) and Brown and Wilson (1956), many alternative explanations for such patterns have been suggested. For example, the pattern might be due to chance, or it might reflect systematic differences between environments where species occur together and where they occur separately. The shifts might be non-genetic and reflect phenotypically plastic changes instead. Or, the mechanism driving divergence might be some other interaction besides resource competition, such as predation or reproductive interference.
Fig. 1 Beak size distributions of ground finches (Geospiza) on several Galápagos islands. Beak sizes of the small ( $G$. fuliginosa) and medium $(G$. fortis) ground finch are more similar where each occurs separately, but are divergent where the species occur together. Redrawn from Lack (1947)

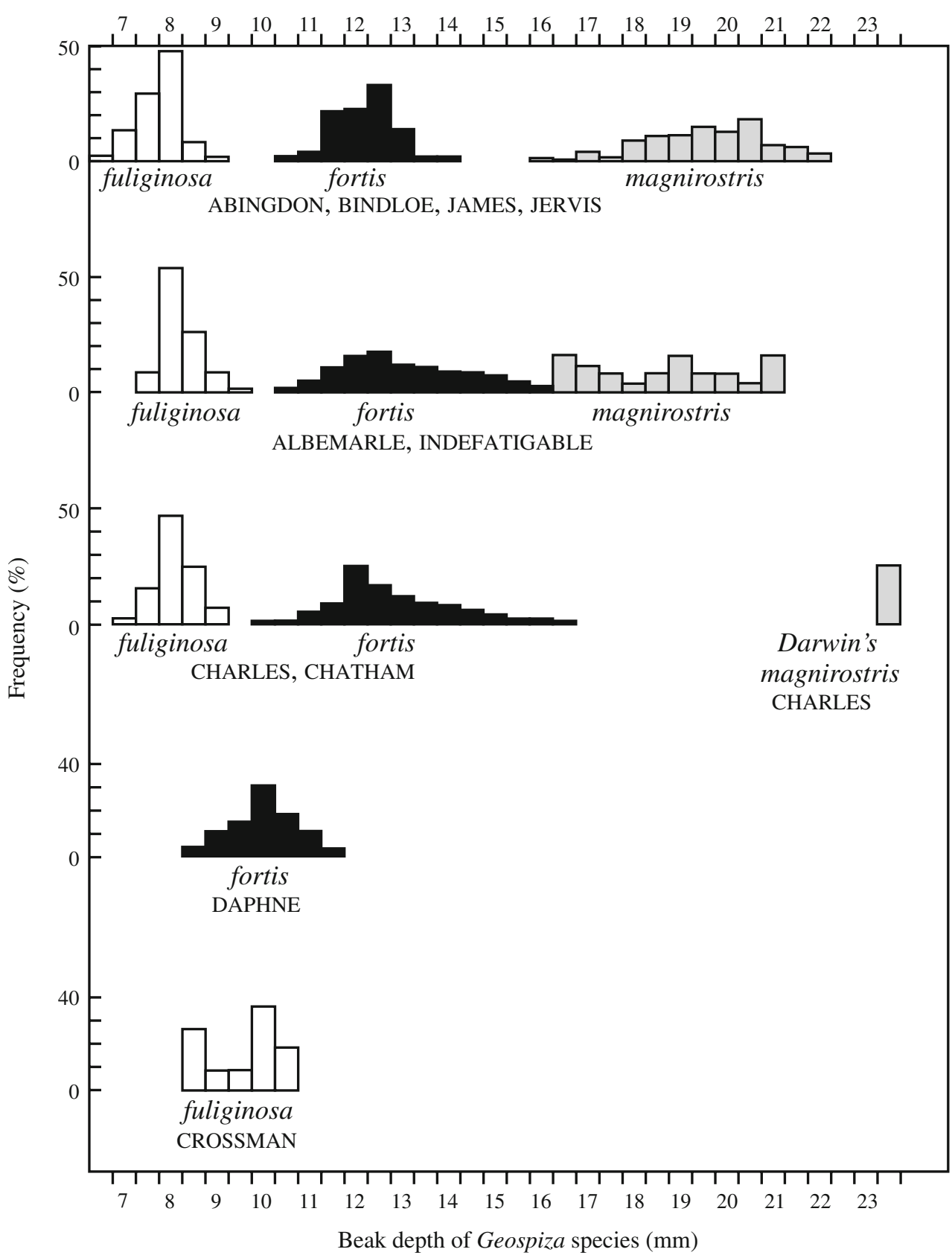


In a few instances, further study found that one or more alternatives to competition provided a superior explanation (Grant 1975; Gorbushin 1996).

For these reasons, evaluating the role of competition and coevolution in the evolution of diversity must go beyond pattern to illuminate the mechanisms driving divergence. Intensive recent research on a few systems, including Darwin's finches (Grant and Grant 2006), spadefoot toads (Pfennig and Murphy 2000), Caribbean island lizards (Losos 2009) and threespine stickleback (Schluter and McPhail 1992; Schluter 2003) have helped to build a more comprehensive picture of the role of competition and coevolution. In this article, I give an overview of progress from studies of one of these groups, the threespine stickleback of small coastal lakes in British Columbia, Canada. These fish show clear patterns like that presented by Lack (1947) on the Galápagos finches, but the stickleback have the additional advantage that it is possible to carry out manipulative experiments.

Our initial aim in the stickleback project was to use experiments to conduct strong tests of the hypothesis of competition and coevolution between coexisting species ("we" and "us" includes myself, students, and collaborators). Along the way we made some unexpected discoveries that indicated both that the effects of competition and coevolution were more far-reaching than we first thought, and at the same time that competition was not the whole story. I highlight some of the still-open questions that these new findings raised, and that will continue to drive research into this system in future.

\section{Patterns of Trait Shift in Threespine Stickleback}

The threespine stickleback in small lakes of coastal British Columbia exhibit a pattern of divergence much like that seen in the Darwin's finches (Fig. 2). Most small lakes contain just one species of threespine stickleback ("solitary" species), but two species occur in a few small lakes on offshore islands (Schluter and McPhail 1992; McPhail 1993). Each coexisting pair includes a "benthic" species that is large and deep-bodied and a "limnetic" species that is smaller and more slender (Fig. 3, photo on left). The two forms are ecologically very distinct. The limnetic feeds mainly on zooplankton in the open water zone of the lake, whereas the benthic eats invertebrates living on or near the vegetation and sediment around the lake margin. Population differences in size and shape persist when fish are raised in the lab, which means that differences between the species are genetically based rather than environmentally induced. Differences between the species in size, shape and other traits ("phenotypic traits") are adaptive, resulting in markedly different abilities to exploit food in the two

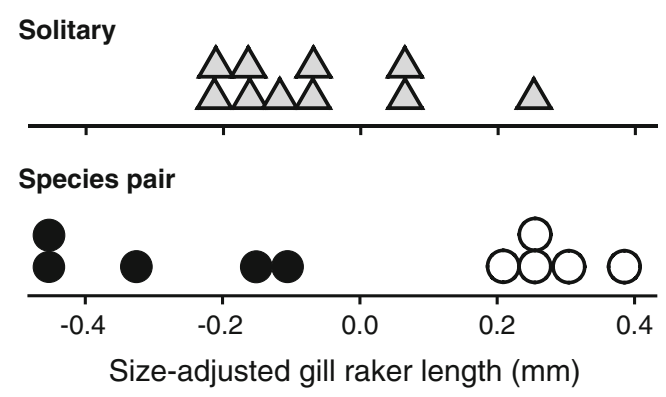

Fig. 2 Population means for paired and solitary threespine stickleback in small coastal lakes of British Columbia, Canada. Gill rakers are filaments on the internal gill arches and are involved in filtering prey from ingested water and directing it to the gut. Gill rakers are generally longer and more numerous in fish that consume zooplankton (such as the limnetic stickleback species) than in fish that consume larger invertebrates or fish. Symbols refer to the benthic (filled circle), limnetic (empty circle), and solitary species. Modified from Schluter (2000), after Schluter and McPhail (1992)

environments of the lakes. Limnetics grow at about twice the rate of benthics when both are placed in enclosures in the offshore environment, where they must feed on zooplankton. Conversely, benthics have a twofold feeding and growth advantage when fish are confined to the shallow, vegetated margins of the lake (Schluter 1993, 1995). These striking phenotypic differences are not found in stickleback species occurring singly in lakes. Such "solitary" stickleback living in otherwise similar lakes are intermediate in size and shape and eat both offshore zooplankton and inshore invertebrates. In other words, phenotypic differences between populations are exaggerated whenever two species have come together in a lake. Figure 2 shows the pattern for just one trait, but the limnetic, benthic, and solitary species are even more distinct when multiple traits are examined together (Schluter and McPhail 1992). A reasonable hypothesis to explain the pattern is that resource competition and coevolution are responsible. In this case, it is likely that there was an initial difference between the ancestors of the benthic and limnetic species that was then amplified in lakes where both occurred. In the next section, I describe how we have tested this idea.

Remarkably, the genetic and geological data indicate that the limnetic and benthic species in different lakes have multiple origins (Taylor and McPhail 1999, 2000). The limnetic species in one lake is not the closest relative to the limnetics in other lakes. The same is true of the benthics. The factors driving divergence between coexisting species are evidently repeatable, and thus the pattern (Fig. 2) cannot be due to chance. These limnetic and benthic species are also among the youngest on earth of any organism, since they occur exclusively in lakes that formed only $10-12,000$ years ago, at the end of the last ice age. The pairs probably evolved from two separate invasions to lakes via the sea (Taylor and 
McPhail 1999, 2000). Coexisting limnetics and benthics are genetically different and reproductively isolated, so we consider them to be distinct species rather than morphs of a single species. However, low levels of gene flow still occur between them within each lake (Gow et al. 2006, 2007).

\section{Testing the Coevolution Hypothesis}

We used experiments to test several predictions of the coevolution hypothesis. The experiments were carried out in a series of ponds on the campus of the University of British Columbia (Fig. 3, photo on right). Each pond was $23 \mathrm{~m} \times 23 \mathrm{~m}$ square with a maximum depth of $3 \mathrm{~m}$ in the center (Schluter 1994). They were constructed in 1991 and seeded with plants and invertebrates from one of the twospecies lakes. The ponds were intended not to be identical to wild lakes but to mimic natural conditions sufficiently well to allow us to test predictions about natural processes. All invertebrates found in the diets of experimental fish were characteristic of the species in the wild. Fish predators of sticklebacks were absent (unless added as part of an experiment) but insect predators of young stickleback were abundant. In the experiments described herein, ponds were divided in two with a plastic membrane, and different treatments were applied to each half. All experiments were short-term, lasting 7-12 weeks within a single stickleback generation. Growth rate was used as a surrogate for fitness, measured by taking the natural log of fish body length (in millimeter) at the end of the experiment.

Experiment 1 The first prediction tested was that divergence, if it had occurred by competition and coevolution, would have yielded lower competition between coexisting species over evolutionary time. To test this, we contrasted the amount of competition experienced by a zooplanktivorous stickleback (in this case the marine species) between two experimental treatments. In one treatment, the zooplanktivore was placed with the benthic species. This treatment represented the present day, two-species lake. In the other treatment, the zooplanktivore was placed with a solitary intermediate species. This treatment approximated the starting point of the stickleback species pairs in lakes, prior to divergence (Schluter and McPhail 1992; McPhail 1993). As predicted by the coevolution hypothesis, the growth rate of zooplanktivorous fish was higher in the benthic species treatment than in the solitary species treatment (Pritchard and Schluter 2001). Stickleback evidently compete for food, and the estimated strength of competition between coexisting species indeed declined after divergence.

Experiment 2 The next prediction tested was that competition from a second stickleback species in a lake should change natural selection on a species already present, and favor divergence. To test it, we contrasted natural selection on an intermediate stickleback between two experimental treatments. In one treatment, the intermediate species was present by itself. In the other treatment a zooplanktivore species (a limnetic) was also added. The target of the experiment was an intermediate stickleback population whose levels of phenotypic variation had been elevated by hybridization to increase the power to detect natural selection (Schluter 1994). The number of individual fish of the intermediate form placed into the ponds was the same between the two treatments, with the consequence that the total number of fish was higher in the treatment with the added zooplanktivore. This design ensured that any differences between treatments in natural selection on the intermediate form could be attributed solely to the added zooplanktivore. As predicted by the coevolution hypothesis, the effects of the added zooplanktivore fell most heavily on those individuals within the target intermediate form that were closest to the added competitor in phenotype and diet (Fig. 4a). Individuals of the intermediate form most different from the added competitor were hardly affected by its presence. Competition indeed generated divergent natural selection between the species.

Experiment 3 The third prediction tested was that natural selection resulting from competition between species should be "frequency dependent." In other words, natural

Fig. 3 On left: adult females of the benthic (upper) and limnetic (lower) species from Paxton Lake, British Columbia. The abdomen of the limnetic female is likely swollen because of an infection by the parasitic cestode, Schistocephalus solidus (photo by T. Hatfield). On right: seining for stickleback in one of the experimental ponds (photo by A. Paccard)

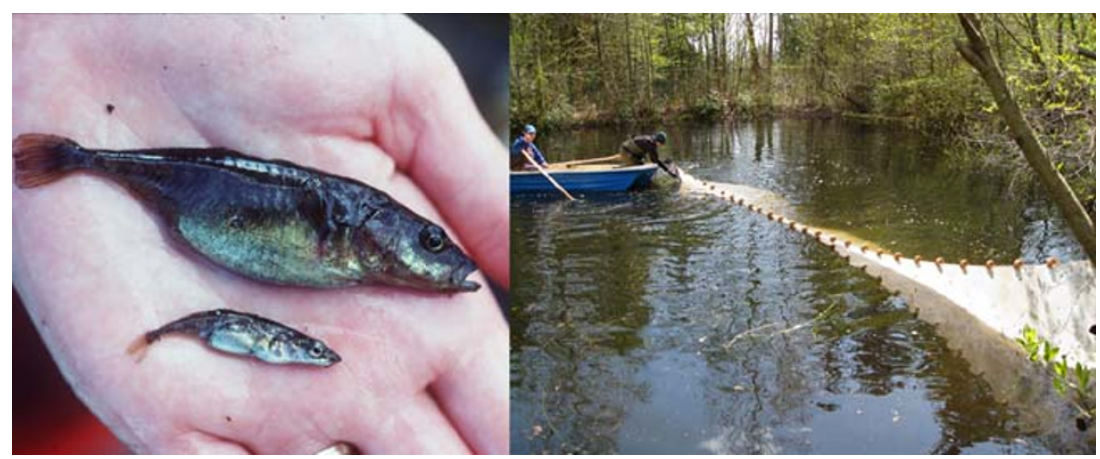


selection pressures should change as the phenotype of the competitor changes. This is an important prediction of the coevolution hypothesis, which proposes that competition and natural selection continue to change as the competitors themselves evolve. The design of the experiment was similar to that of the previous study. In one treatment, the intermediate form was present with a limnetic species. In the other treatment, the intermediate form was present with a benthic species instead. The density of the intermediate form was the same between treatments, but this time total fish densities were also the same. What differed between treatments was the phenotype of the added competitor. As predicted by the coevolution hypothesis, natural selection in the intermediate form differed between treatments (Schluter 2003). In each case, the phenotypes closest to the added competitor felt the greatest impact (Fig. $4 \mathrm{~b}$; we are unable to say whether one competitor had a bigger impact than the other because this experiment didn't include a third treatment in which the intermediate population was present alone). Natural selection arising from competition between species was indeed frequency-dependent.

These three experiments have strongly supported the hypothesis that the observed pattern, in which species are ecologically and phenotypically most different when they coexist (Fig. 3), is the evolutionary outcome of competition for resources. Morphologically more distant populations compete less than more similar forms (experiment 1). Competition from one species generates natural selection on another, favoring divergence (experiment 2). Natural selection arising from competition between species changes depending on the phenotypes of the competitors (experiment 3 ).

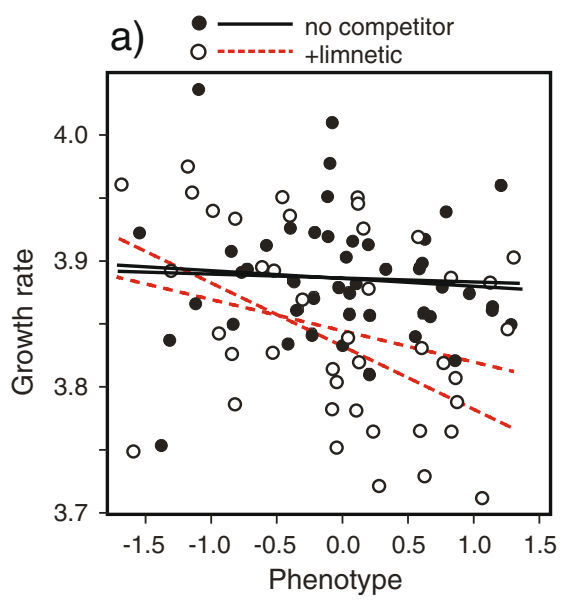

Fig. 4 Growth rates of stickleback differing in phenotype in response to competition. In each panel, the symbols represent stickleback from an experimental intermediate (solitary) population, with more benthiclike individuals on the left and more limnetic-like individuals on the right. Growth rate is measured by ln-transformed fish body length, measured in $\mathrm{mm}$, at the end of the experiment. a Growth rates in the presence (empty circle; dashed lines) and absence (filled circle; solid

\section{Wider Impacts}

The consequences of resource competition between coexisting stickleback do not seem to end with divergence in habitat and resource use. One reason is that offshore and inshore environments also differ in the types of stickleback enemies, and stickleback have adapted to these differences. Stickleback are hunted by cutthroat trout (Oncorhynchus clarki) and diving birds such as loons (Gavia immer), which occur in virtually all the study lakes. These two types of predators are called "gape limited" because they swallow stickleback whole. In addition, young stickleback are preyed upon by insects, especially dragonfly nymphs (Aeshna) and backswimmers (Notonecta), which grab their prey and then chew or suck the fish rather than swallow them whole. Insects are mainly confined to the vegetation near the lake margins, and experiments in wading pools indicate that limnetics are more vulnerable than benthics there (Vamosi 2002). In contrast, the danger to stickleback swimming in open water is mainly from trout and diving birds, and experiments with cormorants suggest that benthics survive worse than limnetics in that environment (Vamosi 2002). Parasites are another source of mortality to stickleback, and the types of parasites also differ between benthics and limnetics (MacColl 2009a).

Bony armor is one adaptation against predators that has diverged between coexisting species. The benthic species consistently has reduced armor compared with limnetics (Vamosi and Schluter 2004) (Fig. 5). Bony lateral plates and spines enhance protection from gape-limited predators, which are the main threat in open water (it makes stickleback more difficult to swallow and improves the

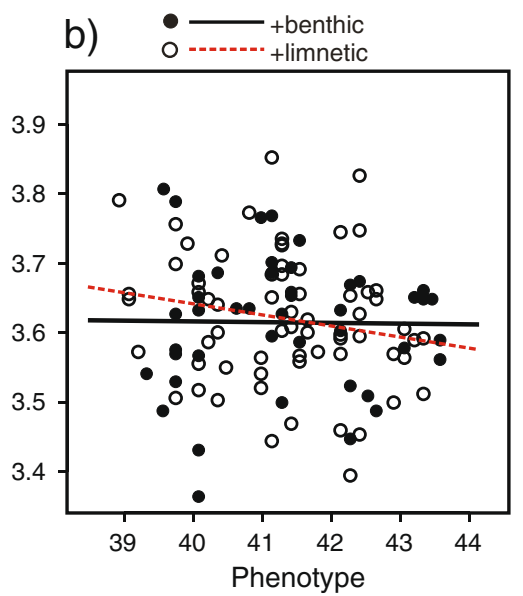

lines) of the limnetic species. Lines represent best fits to data from two replicates of each treatment. Modified from Schluter (1994). b Growth rates in the presence of the limnetic species (empty circle; dashed line) or the benthic species (filled circle; solid line). Data are from one of three replicate ponds; results were similar in the other two. Modified from Rundle et al. (2003). The phenotype axis is scaled differently in the two panels but is otherwise comparable 
chances of survival after escape) (Reimchen 1992, 2000). However, armor is costly to produce (Marchinko and Schluter 2007; Barrett et al. 2009) and might even be a liability inshore where insects are the main killers, possibly by making stickleback easier to grasp (Reimchen 1980; Marchinko 2009). Life history differences may also represent adaptations to contrasting mortality factors. Limnetics are annual species, breeding as one-year-olds and then dying, whereas benthics are perennial, with many not breeding until two years of age (Maccoll 2009b). Higher predation frequently leads to earlier size and age at reproduction (Abrams 1986a).

We're not sure how predation and parasitism interacted with competition to yield divergence between coexisting species. One possibility is that competition was the main cause of divergence in habitat between limnetics and benthics, and that the habitat differences subsequently led to divergence in defensive traits. Under this view the importance of predation and parasitism is secondary to competition. The alternative hypothesis is that predation and parasitism were as vital as competition to divergence between stickleback in habitat, food, and other traits. Sorting out these two possibilities is challenging, but our understanding of the whole process of divergence is at stake. A couple of results so far favor the second of these two hypotheses.

First, solitary populations are not intermediate in armor between limnetics and benthics (Fig. 5), which is unexpected given that they are intermediate in habitat and diet. Instead, solitary stickleback have as much armor as the limnetic species of the two-species lakes. A plausible interpretation of this pattern is that the presence of the limnetic species reduces predation from gape-limited predators on the benthic species, allowing the evolution of reduced armor (Vamosi and Schluter 2004). This interpretation assumes that lakes with one and two stickleback

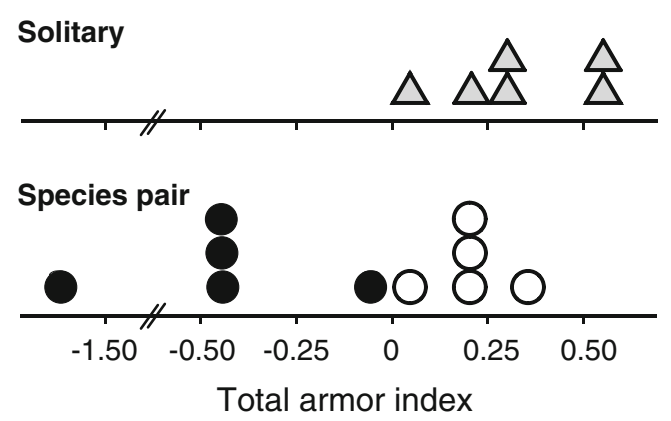

Fig. 5 Population means for paired and solitary threespine stickleback in small coastal lakes of British Columbia, Canada. Total armor is an overall index based on the length of defensive spines and the number of lateral plates. Symbols refer to population means of the benthic (filled circle), limnetic (empty circle), and solitary species. Modified from Vamosi and Schluter (2004) species are not otherwise different in predation and parasitism, which has not been confirmed.

The second result comes from a pond experiment designed to test the effect of predation on competition and divergent selection (Rundle et al. 2003). The design of the experiment was similar to that of experiment 3 described in the previous section, except that we repeated both treatments in two groups of ponds. In one group of ponds, insect predators were depleted (but not eliminated) using traps and nets before the experiment was begun. In the second group of ponds, cutthroat trout and extra insect predators were added to increase total predation on stickleback. Not surprisingly, extra predation increased mortality and reduced (but did not eliminate) competition. Much more surprisingly, the strength of divergent natural selection between competitors was increased rather than diminished in ponds where mortality was high. This result seems paradoxical, but the strength of divergent selection is expected to depend not so much on how strong competition is, but rather on how rapidly competition declines between individuals as they differ more greatly in phenotype (Rundle et al. 2003). Abrams et al. (2008) has identified the conditions under which predation strengthens or weakens divergent selection from competition. In our experiment, at least, predation promoted divergence via competition.

\section{Coevolution and Speciation}

Finally, there is indication that coevolution has contributed to the process of stickleback speciation - the origin of the limnetic and benthic species pairs.

Speciation is defined as the evolution of reproductive isolation (reduced gene exchange) between populations (Coyne and Orr 2004).

An extraordinary aspects of the stickleback species pairs is their youth - they occur only in lakes that formed 10 12,000 years old. Like most very young species occurring together, limnetic and benthic stickleback species interbreed to a small degree, yet this has not caused their collapse under normal circumstances. Hybrids are selected against (Gow et al. 2007), and one reason is that they are morphologically intermediate and thus have a lower feeding efficiency and growth rate in both the inshore and offshore habitats compared with the limnetic and benthic species (Hatfield and Schluter 1999; Rundle 2002). Hybrids are at a competitive disadvantage. It is likely that this competitive disadvantage is greater today than earlier in the history of the species pairs, because each new adaptation in the benthic or limnetic species that improved its ability to exploit its preferred habitat would have further reduced the fitness of intermediate phenotypes, including hybrids. The 
implication is that competition and coevolution, by facilitating phenotypic divergence, have contributed to the reduction of gene flow between them and to their persistence over time.

This argument considers only the fate of hybrids, but in fact not many hybrids are formed because of behavioral differences between the species. Limnetics strongly prefer to mate with limnetics, and benthics mate almost exclusively with benthics. We now know that body size is one of the cues used by stickleback to identify and mate with their own type (Nagel and Schluter 1998). Size is a fairly effective cue because large, reproductively mature benthics hardly overlap the smaller limnetics in size. In this case, it is easy to see how natural selection, by favoring different body sizes in the inshore and offshore environments, would indirectly strengthen the tendency of limnetics to mate with limnetics, and benthics with benthics (Vines and Schluter 2006). This represents a second way in which competition, by favoring divergence, would contribute to reduced gene exchange. Such an influence of competition on speciation is something that Darwin (1859) foresaw, but for which evidence is only now beginning to emerge.

\section{Conclusions}

The threespine stickleback provides observational and experimental evidence for competition and coevolution in the divergence of coexisting species. Our studies of this system have also suggested that other interactions, such as predation and parasitism, have played an important part. Finally, there is evidence that divergent selection arising from these interactions has facilitated the very origin of species. The stickleback species pairs represent a powerful case study of the influence of coevolution on the generation of biodiversity.

Nevertheless, questions remain that continue to demand attention. One challenge is to connect measurements of natural selection on phenotypic traits to genetic changes underlying evolved differences between the species. Such studies are only now becoming feasible with the availability of complete genome sequences for stickleback (Kingsley et al. 2004), and with the identification of major genes and genomic regions underlying species differences. For example, knowing something about the genes will make it possible to carry out experimental studies to measure the effects of competition and other species interactions at the molecular level. For these reasons, research on the stickleback species pairs will continue to provide insights into the coevolutionary process.

All these efforts to understand the impacts of coevolution on divergence and speciation in stickleback would justify only mild curiosity if the results turned out to be unique to this system. However, intensive studies of young species in other groups of organisms including Anolis lizards on Caribbean islands (Losos 2009), spadefoot toad tadpoles in the American southwest (Pfennig and Murphy 2000), and Darwin's finches on Galápagos islands (Grant and Grant 2006), have uncovered similar mechanisms, suggesting that they occur widely (though not necessarily universally). Competition and coevolution are likely to be major drivers of species differentiation in nature. Without them, many closely related species would be more similar in ecology and phenotype, and the breadth of life's diversity would likely be considerably less.

Acknowledgments Our work was made possible by the discovery and early descriptions of the stickleback species pairs by Don McPhail and his students. I am also grateful to the University of British Columbia for supporting the experimental ponds facility. Thanks to John Thompson and Rodrigo Medel for the invitation to participate in this issue, and for their comments on the manuscript. My research is funded by the Natural Sciences and Engineering Research Council of Canada, the British Columbia Knowledge Development Fund, the Canada Foundation for Innovation, and the Canada Research Chairs.

\section{References}

Abrams PA. Adaptive responses of predators to prey and prey to predators: the failure of the arms race analogy. Evolution. 1986a;40:1229-47.

Abrams PA. Character displacement and niche shift analyzed using consumer-resource models of competition. Theor Popul Biol. 1986b;29:107-60.

Abrams PA, Rueffler C, Kim G. Determinants of the strength of disruptive and/or divergent selection arising from resource competition. Evolution. 2008;62:1571-86.

Barrett RDH, Rogers SM, Schluter D. Environment specific pleiotropy facilitates divergence at the Ectodysplasin locus in threespine stickleback. Evolution. 2009;63:2831-7.

Brown Jr WL, Wilson EO. Character displacement. Syst Zool. 1956;5:49-64.

Coyne JA, Orr HA. Speciation. Sunderland: Sinauer Associates; 2004.

Darwin C. On the origin of species by means of natural selection. London: John Murray; 1859.

Doebeli M. An explicit genetic model for ecological character displacement. Ecology. 1996;77:510-20.

Gorbushin AM. The enigma of mud snail shell growth: asymmetrical competition or character displacement? Oikos. 1996;77:85-92.

Gow JL, Peichel CL, Taylor EB. Contrasting hybridization rates between sympatric three-spined sticklebacks highlight the fragility of reproductive barriers between evolutionarily young species. Mol Ecol. 2006;15:739-52.

Gow JL, Peichel CL, Taylor EB. Ecological selection against hybrids in natural populations of sympatric threespine sticklebacks. J Evol Biol. 2007;20:2173-80.

Grant PR. The classic case of character displacement. Evol Biol. 1975;8:237-337.

Grant PR, Grant BR. Evolution of character displacement in Darwin's finches. Science. 2006;313:224-6.

Grant PR, Grant BR. How and why species multiply: the radiation of Darwin's finches. Princeton: Princeton University Press; 2008. 
Hatfield T, Schluter D. Ecological speciation in sticklebacks: environment-dependent hybrid fitness. Evolution. 1999;53:866-73.

Kingsley DM, Zhu BL, Osoegawa K, De Jong PJ, Schein J, Marra M, et al. New genomic tools for molecular studies of evolutionary change in threespine sticklebacks. Behaviour. 2004;141:1331-44.

Lack D. Darwin's finches. Cambridge: Cambridge University Press; 1947.

Losos JB. Lizards in an evolutionary tree: the ecology of adaptive radiation in anoles. Berkeley: University of California Press; 2009.

MacColl ADC. Parasite burdens differ between sympatric three-spined stickleback species. Ecography. 2009a;32:153-60.

Maccoll ADC. Parasites may contribute to 'magic trait' evolution in the adaptive radiation of three-spined sticklebacks, Gasterosteus aculeatus (Gasterosteiformes: Gasterosteidae). Biol J Linn Soc. 2009b;96:425-33.

Marchinko KB. Predation's role in repeated phenotypic and genetic divergence of armor in threespine stickleback. Evolution. 2009;63:127-38.

Marchinko KB, Schluter D. Parallel evolution by correlated response: lateral plate reduction in threespine stickleback. Evolution. 2007;61:1084-90.

McPhail JD. Ecology and evolution of sympatric sticklebacks (Gasterosteus): origin of the species pairs. Can J Zool. 1993;71:515-23.

Nagel L, Schluter D. Body size, natural selection, and speciation in sticklebacks. Evolution. 1998;52:209-18.

Pfennig DW, Murphy PJ. Character displacement in polyphenic tadpoles. Evolution. 2000;54:1738-49.

Pritchard JR, Schluter D. Declining interspecific competition during character displacement: summoning the ghost of competition past. Evol Ecol Res. 2001;3:209-20.

Reimchen TE. Spine deficiency and polymorphism in a population of Gasterosteus aculeatus: an adaptation to predators? Can J Zool (Revue Canadienne De Zoologie). 1980;58:1232-44.

Reimchen TE. Injuries on stickleback from attacks by a toothed predator (Oncorhynchus) and implications for the evolution of lateral plates. Evolution. 1992;46:1224-30.
Reimchen TE. Predator handling failures of lateral plate morphs in Gasterosteus aculeatus: Functional implications for the ancestral plate condition. Behaviour. 2000;137:1081-96.

Rundle HD. A test of ecologically dependent postmating isolation between sympatric sticklebacks. Evolution. 2002;56:322-9.

Rundle HD, Vamosi SM, Schluter D. Experimental test of predation's effect on divergent selection during character displacement in sticklebacks. Proc Natl Acad Sci USA. 2003;100:14943-8.

Schluter D. Adaptive radiation in sticklebacks: size, shape, and habitat use efficiency. Ecology. 1993;74:699-709.

Schluter D. Experimental evidence that competition promotes divergence in adaptive radiation. Science. 1994;266:798-801.

Schluter D. Adaptive radiation in sticklebacks: trade-offs in feeding performance and growth. Ecology. 1995;76:82-90.

Schluter D. The ecology of adaptive radiation. Oxford: Oxford University Press; 2000.

Schluter D. Frequency dependent natural selection during character displacement in sticklebacks. Evolution. 2003;57:1142-50.

Schluter D, McPhail JD. Ecological character displacement and speciation in sticklebacks. Am Nat. 1992;140:85-108.

Taper ML, Case TJ. Quantitative genetic models for the coevolution of character displacement. Ecology. 1985;66:355-71.

Taylor EB, McPhail JD. Evolutionary history of an adaptive radiation in species pairs of threespine sticklebacks (Gasterosteus): insights from mitochondrial DNA. Biol J Linn Soc. 1999;66:271-91.

Taylor EB, McPhail JD. Historical contingency and ecological determinism interact to prime speciation in sticklebacks, Gasterosteus. Proc R Soc Lond B. 2000;267:2375-84.

Thompson JN. The geographic mosaic of coevolution. Chicago: University of Chicago Press; 2005.

Vamosi SM. Predation sharpens the adaptive peaks: survival trade-offs in sympatric sticklebacks. Ann Zool Fenn. 2002;39:237-48.

Vamosi SM, Schluter D. Character shifts in the defensive armor of sympatric sticklebacks. Evolution. 2004;58:376-85.

Vines TH, Schluter D. Strong assortative mating between allopatric sticklebacks as a by-product of adaptation to different environments. Proc R Soc Lond B. 2006;273:911-6. 